\title{
Remodelação cirúrgica da valva aórtica
}

\author{
Ronaldo D. FONTES*, Herbert D. SALERNO*, Alfredo V. E. E. HIJO*, Alberto NAJJAR*, \\ Luiz César N. SCALA*, Maria Cecília K. FARAH*, Gustavo J. V. COUTO*
}

RBCCV 44205-549

Fontes R D, Salerno H D, Hijo A V E E, Najjar A, Scala L C N, Farah M C K, Couto G J V - Remodelação cirúrgica da valva aórtica. Rev Bras Cir Cardiovasc 2001; 16(3): 212-17.

RESUMO: Objetivo: Os autores analisam os resultados por eles obtidos com a utilização de técnica específica para a preservação da valva aórtica.

Casuística e Métodos: Esse método consiste no emprego habitual da circulação extracorpórea com uso de hipotermia moderada e infusão de solução cardioplégica nos óstios coronários. Realizase ressecção da válvula não coronariana e une-se as comissuras correspondentes utilizando-se fio de Mersilene 2-0 ancorado em feltro de teflon, tornando a valva "bivalvulada". Procede-se por fim à aortorrafia convencional. Foram operados 15 pacientes, 9 do sexo masculino. A idade variou entre 12 e 78 anos. Quatro pacientes tinham diagnóstico de insuficiência aórtica isolada, 2 com dupla disfunção aórtica associada à insuficiência coronária, 2 com doença da aorta ascendente, 4 com insuficiência aórtica e mitral, 1 com dupla disfunção aórtica e mitral, e 2 com insuficiência aórtica e insuficiência coronária.

Resultados: Em 12 pacientes não houve insuficiência aórtica residual pós-operatória, e em 3 identificou-se insuficiência aórtica leve ou moderada. Três pacientes foram reoperados no pósoperatório tardio devido a insuficiência aórtica, tendo sido substituída a valva aórtica em 2. Quatro pacientes foram submetidos a estudo hemodinâmico e em 1 deles havia gradiente supravalvar de $20 \mathrm{mmHg}$. Nos outros 3 pacientes, o gradiente sistólico transvalvar foi de 0,9 e $12 \mathrm{mmHg}$, respectivamente. Os 11 pacientes restantes foram avaliados somente através de ecocardiografia, a qual não demonstrou estenose. Não houve óbito imediato e houve 1 óbito tardio, após reoperação. Quatorze pacientes tiveram melhora da classe funcional, tanto imediata quanto tardiamente, e foram acompanhados durante período entre 30 dias e 24 meses, encontrando-se em classe funcional I ou II (NYHA).

Conclusão: Os autores acreditam que o método seja uma alternativa para o tratamento cirúrgico conservador da doença valvar aórtica.

DESCRITORES: Valva aórtica, cirurgia. Insuficiência da valva aórtica, cirurgia. Insuficiência da valva aórtica, cirurgia, seguimentos.

Trabalho realizado no Hospital Santa Cruz - Serviço de Cirurgia Cardiovascular R. D. Fontes. Cuiabá, MT, Brasil.

Recebido para publicação em fevereiro de 2000.

* Do Hospital Santa Cruz - Serviço de Cirurgia Cardiovascular R. D. Fontes.

Endereço para correspondência: Ronaldo D. Fontes. Av. Lavapés, 212 , sala 701. Cuiabá, MT. CEP: 78020-870. Tel: (65) 622-2036. e-mail: ronaldofontes@terra.com.br 
Fontes R D, Salerno H D, Hijo A V E E, Najjar A, Scala L C N, Farah M C K, Couto G J V - Remodelação cirúrgica da valva aórtica. Rev Bras Cir Cardiovasc 2001; 16(3): 212-17.

\section{INTRODUÇÃO}

O tratamento cirúrgico conservador da valva aórtica em pacientes com insuficiência valvar aórtica não tem resultado tão satisfatório quanto o da insuficiência mitral (1). Entretanto, as tentativas de preservação cirúrgica da valva aórtica são, na realidade, expressão das complicações que as próteses cardíacas valvares apresentam em sua evolução, tanto pelas limitações impostas aos pacientes que recebem próteses mecânicas, como pela possibilidade de reoperação para troca de prótese biológica que sofre processo degenerativo(2).

Assim sendo, métodos empregados na tentativa de preservação da valva aórtica devem ser considerados, padronizados e analisados a longo prazo. É o caso do presente artigo, que tem por objetivo relatar resultado de técnica de preservação da valva aórtica, utilizada primeiramente por GARAMELLA et al. (3), em 1958, e, posteriormente, abandonada.

\section{CASUÍSTICA E MÉTODOS}

No período compreendido entre março de 1996 e julho de 1999 foram operados 15 pacientes portadores de disfunção valvar aórtica em nosso Serviço.

Quatro doentes tinham diagnóstico de insuficiência aórtica isolada, 2 com dupla disfunção aórtica associada à insuficiência coronária, 2 com doença da aorta ascendente, $4 \mathrm{com}$ insuficiência aórtica e mitral, $1 \mathrm{com}$ dupla disfunção aórtica e mitral, e 2 com insuficiência aórtica e insuficiência coronária. A idade dos pacientes variou entre 12 e 78 anos, sendo que 2 deles tinham idade inferior a 15 anos. Nove pacientes eram do sexo masculino.

O método empregado na correção cirúrgica da valvopatia aórtica foi denominado "Remodelação Cirúrgica da Valva Aórtica" e, conforme descrição de GARAMELLA et al. (3), consiste em utilização habitual de circulação extracorpórea, hipotermia moderada e infusão de solução cardioplégica nos óstios coronários. A válvula não coronariana é ressecada e é passado ponto ancorado nas comissuras correspondentes à válvula ressecada, de maneira que quando tracionado, torna a valva aórtica bivalvulada. O seio de Valsalva correspondente à válvula ressecada fica situado abaixo da "nova comissura". A parede da aorta torna-se evaginada, sendo suas bordas suturadas com monofilamento 5-0, continuamente, prosseguindo-se com a aortorrafia convencional (Figura 1).

\section{RESULTADOS}

Dos 15 pacientes operados no período, 6 foram submetidos à remodelação cirúrgica da valva aórtica como descrita no método, 2 foram tratados com remodelação e interposto enxerto tubular de pericárdio bovino, substituindo a aorta ascendente, outros 2 com remodelação da valva aórtica associada à revascularização do miocárdio, $1 \mathrm{com}$ remodelação mais comissurotomia mitral e aórtica e os 4 últimos submetidos à remodelacão da valva aórtica e plástica da valva mitral (Tabela 1). Não houve óbito imediato. O tempo de circulação extracorpórea variou entre 47 e 122 minutos e o tempo de anóxia miocárdica entre 18 e 100 minutos.

Todos os pacientes tiveram boa evolução no pós-operatório imediato (30 dias), melhorando sua classe funcional, entretanto, 3 deles ficaram com insuficiência aórtica leve ou moderada após o terceiro ou quarto dias de pós-operatório. A pressão arterial, medida através de esfigmomanômetro no pré-operatório, foi comparada com o pós-operatório, e revelou níveis praticamente normais em todos os pacientes, imediatamente após a operação.

Os pacientes foram acompanhados em período pós-operatório tardio que variou entre 30 dias e 24 meses.

Quatro pacientes foram submetidos a estudo hemodinâmico no pós-operatório imediato. Em um deles não houve gradiente transvalvar aórtico, e, nos outros 3 pacientes, os gradientes encontrados foram de 9,12 e $20 \mathrm{mmHg}$, este último supravalvar. Nenhum dos 4 pacientes tinha insuficiência aórtica ao exame (Figura 2).

Três (20\%) pacientes desenvolveram insuficiência aórtica no pós-operatório, tendo sido necessária reintervenção cirúrgica. Um dos pacientes, com diagnóstico de ectasia ânulo-aórtica associada à insuficiência aórtica, evoluiu no pósoperatório imediato com insuficiência aórtica leve/moderada. Este paciente foi submetido ao teste de esforço (protocolo de Bruce), suportou carga máxima e conseguiu terminar o exame, portanto em classe funcional I (NYHA). Recebeu alta em boas condições clínicas, com pressão arterial de $130 \times 60 \mathrm{mmHg}$. Após 10 meses, evoluiu com dispnéia aos médios esforços, dor precordial e pressão arterial de $180 \times 30 \mathrm{mmHg}$, sendo reoperado para troca da valva aórtica. Um segundo paciente, portador de dupla disfunção aórtica e insuficiência coronária, que tinha sido submetido a remodelação e revascularização do miocárdio, apresentou insuficiên- 
Fontes R D, Salerno H D, Hijo A V E E, Najjar A, Scala L C N, Farah M C K, Couto G J V - Remodelação cirúrgica da valva aórtica. Rev Bras Cir Cardiovasc 2001; 16(3): 212-17.

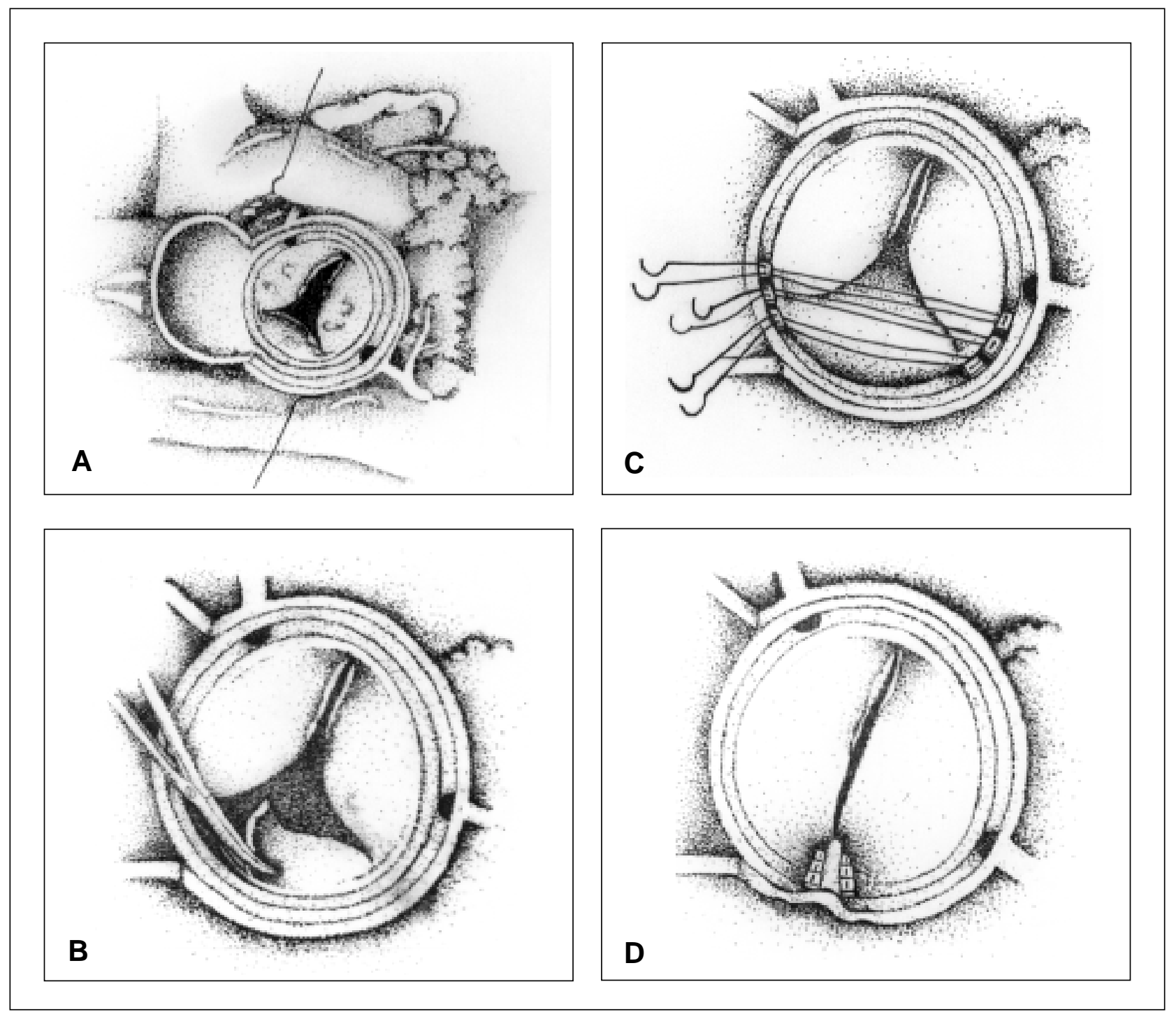

Fig. 1 - A. Desenho que representa a valva aórtica competente; B. Ressecção da válvula aórtica não coronariana; C. Pontos ancorados em feltro de teflon são passados junto às comissuras correspondentes à válvula ressecada. D. Observa-se no aspecto final do esquema da operação, a valva aórtica "bicuspidizada".

cia aórtica importante no segundo mês de pósoperatório. Este paciente foi submetido a nova intervenção cirúrgica, tendo sido trocada a valva aórtica. Havia rotura da sutura e a plicatura do anel estava solta. Evoluiu com distúrbio neurológico no pós-operatório tardio, falecendo. O terceiro paciente, um menino de 14 anos de idade, com diagnóstico de insuficiência aórtica pura, foi submetido a remodelação isolada e evoluiu com insuficiência aórtica importante após 3 meses de pós-operatório. O menor foi reoperado e encontrada rotura da plicatura da parede aórtica, provocando insuficiência aórtica paravalvar. Optou-se novamente pela conservação da valva aórtica motivada pelo bom diâmetro do anel e pelo aspecto normal das válvulas remanescentes. Porém, continua com insuficiência aórtica moderada, em acompanhamento clínico.

Durante o período de seguimento tardio, todos os pacientes estão em classe funcional (NYHA) melhor do que no pré-operatório. Dois deles têm insuficiência aórtica mínima e apenas um moderada. Não houve óbito imediato, apenas um tardio entre três que foram reoperados para tratar insuficiência aórtica. 
Fontes R D, Salerno H D, Hijo A V E E, Najjar A, Scala L C N, Farah M C K, Couto G J V - Remodelação cirúrgica da valva aórtica. Rev Bras Cir Cardiovasc 2001; 16(3): 212-17.

TABELA 1

\begin{tabular}{|c|c|c|c|c|c|c|c|}
\hline \multicolumn{8}{|c|}{ DIAGNÓSTICO, PROCEDIMENTO E A EVOLUÇÃO PÓS-OPERATÓRIA } \\
\hline IDADE & SEXO & DIAGNÓSTICO & OPERAÇÃO & $\begin{array}{l}\text { EVOLUÇÃO } \\
\text { IMEDIATA }\end{array}$ & $\begin{array}{c}\text { ÓBITO } \\
\text { IMEDIATO }\end{array}$ & $\begin{array}{c}\text { EVOLUÇÃO } \\
\text { TARDIA }\end{array}$ & $\begin{array}{r}\text { ÓBITO } \\
\text { TARDIO }\end{array}$ \\
\hline 12 & Masculino & IAO & remd. & Sem complicações & Não & Sem complicações & Não \\
\hline 14 & Masculino & IAO & remd. & Sem complicações & Não & IAO reop re md. lao & Não \\
\hline 56 & Masculino & IAO+dis.ao. & remd.+prao & IAO moderada & Não & IAO reop tvao & Não \\
\hline 58 & Masculino & dlao+ico & remd.+rm & Sem complicações & Não & Sem complicações & Não \\
\hline 63 & Masculino & dlao+ico & remd.+rm & Sem complicações & Não & IAO reop coma & Sim \\
\hline 48 & Masculino & $\mathrm{IAO+aneao}$ & remd.+prao & IAO discreta & Não & Sem complicações & Não \\
\hline 72 & Feminino & $\mathrm{IAO+ico}$ & remd.+rm & Sem complicações & Não & Sem complicações & Não \\
\hline 78 & Masculino & $\mathrm{IAO}+\mathrm{ico}$ & remd.+rm & Sem complicações & Não & Sem complicações & Não \\
\hline 37 & Feminino & IAO+imi & remd. + plmi & Sem complicações & Não & Sem complicações & Não \\
\hline 43 & Feminino & $\mathrm{IAO}+\mathrm{imi}$ & remd. + plmi & Sem complicações & Não & Sem complicações & Não \\
\hline 54 & Feminino & $I A O+i m i$ & remd. + plmi & Sem complicações & Não & Sem complicações & Não \\
\hline 17 & Masculino & $I A O+i m i$ & remd. + plmi & Sem complicações & Não & Sem complicações & Não \\
\hline 72 & Masculino & dlao + dlmi & remd. + com & Sem complicações & Não & Sem complicações & Não \\
\hline 61 & Feminino & IAO & remd & Sem complicações & Não & Sem complicações & Não \\
\hline 40 & Feminino & $\mathrm{IAO}$ & remd & Sem complicações & Não & Sem complicações & Não \\
\hline
\end{tabular}

IAO = insuficiência aórtica; remd = remodelação; reop = reoperação; dis. ao = dissecção crônica da aorta; prao = interposição de prótese em aorta ascendente; tvao = troca da valva aórtica; aneao = aneurisma de aorta ascendente; plmi = plástica da valva mitral; com = comissurotomia mitral e aórtica

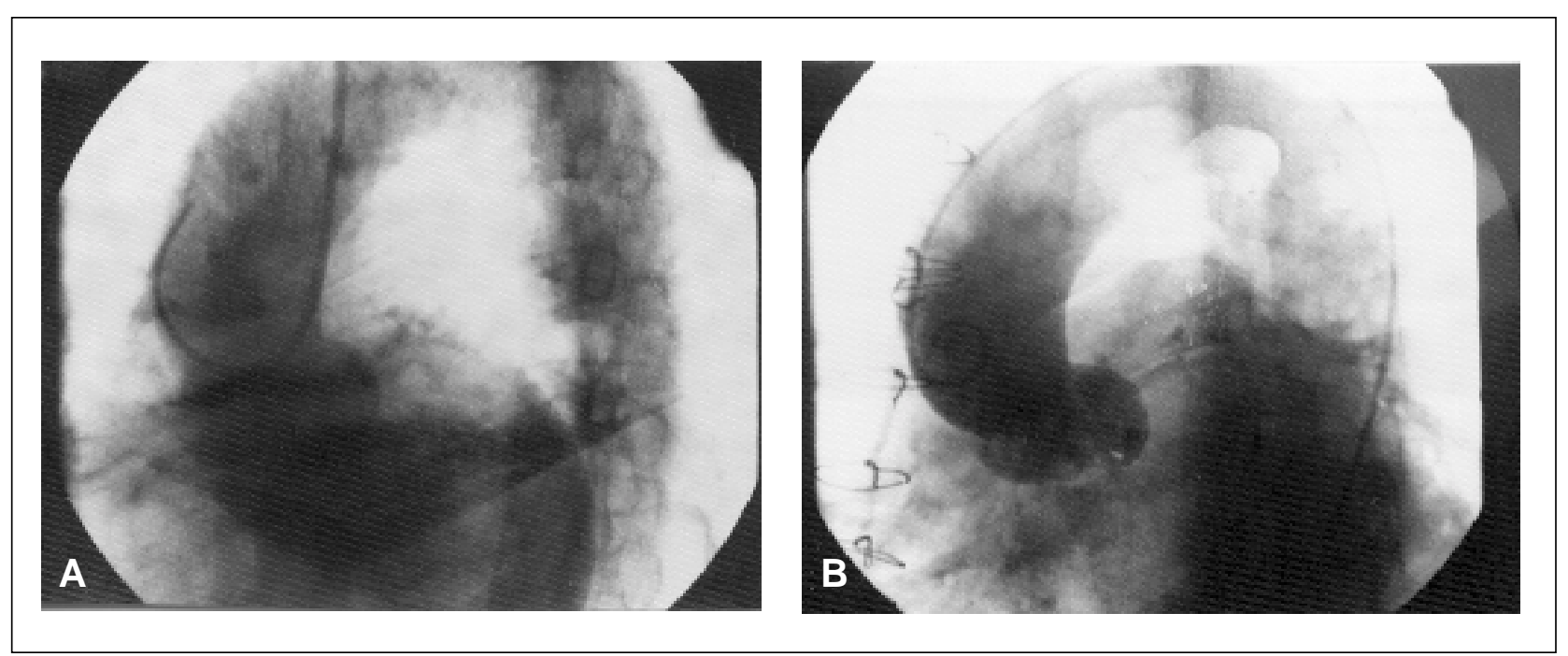

Fig. 2 - A. Aortografia pré-operatória, demonstrando insuficiência da valva aórtica. B. Aortografia pós-operatória na qual observamos competência da valva aórtica, elevação do plano valvar ao nível da nova comissura e diminuição do diâmetro da aorta ascendente ao nível do plano das comissuras. 


\section{COMENTÁRIOS}

As características morfológicas da insuficiência aórtica dependem basicamente da etiologia do processo degenerativo que acomete a valva. A doença reumática, responsável por aproximadamente $46 \%$ dos casos de insuficiência aórtica isolada, provoca calcificação em apenas $10 \%$ dos casos e geralmente é focal. Mais freqüentemente, a doença reumática provoca retração que com o passar do tempo, leva a insuficiência central da valva aórtica ${ }^{(1,2)}$.

A ectasia ânulo-aórtica produz vários graus de insuficiência aórtica, por vezes importante, entretanto suas válvulas são consideradas normais(4). Acredita-se que a doença cística da camada média cause dilatação da parede aórtica junto aos seios de Valsalva, e estendendo-se à aorta proximal, afaste as comissuras da valva aórtica, provocando insuficiência $(1,4)$. A ectasia ânulo-aórtica, com ou sem dissecção, é responsável por aproximadamente 18\% dos casos de insuficiência aórtica (4).

Dentre as etiologias mais freqüentes da insuficiência aórtica, podemos destacar também a doença valvar congênita, que ocorre em $13 \%$ dos casos, representada morfologicamente pela presença de valva aórtica bi ou univalvulada(1). Essas alterações podem provocar insuficiência aórtica por prolapso, serem agravadas pela ocorrência de endocardite ou comissurotomia inadequada (1).

Outras etiologias menos freqüentes perfazem aproximadamente $20 \%$ dos casos de insuficiência aórtica como endocardite nativa, doenças mixomatosas, aterosclerose, sífilis, aortites, rotura espontânea ou pós-trauma (1-5). Classicamente, o tratamento da insuficiência aórtica é a troca valvar por prótese artificial, biológica ou mecânica (1).

Várias técnicas e tipos de próteses valvares têm sido empregados para esse fim, desde 1957, quando Hufnagel implantou a primeira "prótese de bola" em aorta descendente (1). Atualmente, a maioria dos centros utiliza prótese mecânica com resultados favoráveis ${ }^{(5)}$. Entretanto, o tromboembolismo ocorre, ainda que, em algumas séries, com freqüência baixa $(6,7)$.

As próteses biológicas têm sido uma opção bastante aceitável, mesmo levando-se em conta a deterioração que ocorre nos tecidos empregados para sua confecção ${ }^{(1,7)}$. Devido às complicações decorrentes desses processos degenerativos que acometem as biopróteses, alguns autores recorrem às técnicas de preservação da valva aórtica(2-4), inclusive, em alguns casos, à bicuspidização(8-9), utilizada inicialmente entre 1958 e 1960 por GARAMELLA et al.(3), STARZL et al. (10), HURWITT et al. (11) revista, em 1966, por CABROL et al.(12), e, posteriormente, abandonada.

As técnicas atuais incluem plicatura das comissuras, redução do anel aórtico através de suturas, excisão de porção de uma ou mais válvulas redundantes e reconstrução por sutura (2-4,8,9).

Entretanto, as indicações e os resultados obtidos com essas técnicas ainda são inconclusivos. Como sabemos, as diversas etiologias pertinentes à insuficiência aórtica têm características distintas quanto a sua morfologia, e talvez possam ser utilizadas como critérios de indicação para preservação da valva aórtica. A técnica descrita neste trabalho foi por nós utilizada no primeiro paciente por existirem condições anatômicas favoráveis. A válvula não coronariana era menor que as outras duas, que tinham aspecto normal. Além disso, o anel aórtico tinha bom diâmetro. Em outro paciente havia dupla disfunção aórtica e calcificação de suas três válvulas. Após a descalcificação das mesmas, foi possível realizar a "Remodelação Cirúrgica da Valva Aórtica" com muita facilidade. O terceiro paciente tinha diagnóstico de ectasia ânulo-aórtica, sendo a redução do anel facilitada pelo seu maior diâmetro. Esse paciente apresentou insuficiência aórtica leve no terceiro dia de pós-operatório e no quinto dia evoluiu para insuficiência aórtica moderada causada por escape de ponto de sutura da aorta ascendente, conforme comprovado cirurgicamente quando foi submetido a troca valvar aórtica. Não houve óbito imediato nesta série de pacientes operados, nem ocorreram complicações extracardíacas. Apenas um paciente ficou com gradiente transvalvar de 20 $\mathrm{mmHg}$. Entretanto, uma vez que esse paciente tinha 12 anos de idade e existindo tecido valvar nativo, é possível que as estruturas cresçam diminuindo o gradiente. Não houve insuficiência aórtica residual (Figura 1).

A insuficiência aórtica, que ocorreu em 3 casos por rotura de pontos, terá que ser resolvida, talvez com pontos da plicatura passados externamente à parede aórtica e ancorados em feltro de teflon.

Em conclusão, os autores acreditam que o método possa ser reproduzido com sucesso para alguns pacientes com valvopatia aórtica. 
Fontes R D, Salerno H D, Hijo A V E E, Najjar A, Scala L C N, Farah M C K, Couto G J V - Surgical remodeling of the aortic valve. Rev Bras Cir Cardiovasc 2001; 16(3): 212-17.

ABSTRACT: Purpose: The authors report a method of surgical treatment of the aortic valve disease, called "Surgical Remodeling of Aortic Valve".

Material and Method: The method consisted of the utilization of the habitual extracorporeal circulation, moderated hipothermia and the infusion of cardioplegic solution in the coronary ostia. The non-coronary valvula is resected and stitches anchored in the aortic valva anulus are given in a way to approximate the resected valvula commissures, in order to turn the trivalvular valva into a bivalvulate one. The Valsalva sinus corresponding to the resected valva stays situated below the commissure and the remaining aortic wall is sutured with a few separated stitches, followed with the conventional aortorraphy. Between March of 1996 and July of 1999, 15 patients were operated with the described technique. Nine were male and the age ranged from 12 to 78 years. Four patients had aortic valve insufficiency, 4 with aortic and mitral insufficiency, 2 with double aortic lesion and coronary artery disease, 2 with ascending aortic disease, 2 with aortic insufficiency and coronary artery disease, and 1 with double dysfunction of aortic and mitral valves.

Results: Three patients developed aortic insufficiency on the post-operative and 3 of them were operated on in the late post-operative. Four patients were submitted to hemodynamic study and one of them had a supravalvar gradient estimated at $20 \mathrm{mmHg}$. Eleven patients were studied by echocardiogram and did not have stenosis. There were neither immediate obits and one occurred in the late post-operative period. Fourteen patients were followed during 30 days to 24 months, and had a good evolution (Functional Class I or II of the NYHA).

Conclusion: This method for the aortic valve preservation is an alternate option for the surgical treatment of the aortic valve disease.

DESCRIPTORS: Aortic valve, surgery. Aortic valve insufficiency, surgery. Aortic valve insufficiency, surgery, follow-up studies.

\section{REFERÊNCIAS BIBLIOGRÁFICAS}

1 Kirklin J \& Barrat-Boyes B - Cardiac surgery. Second Edition. New York: Churchill-Livingstone Inc, 1993: 560 .

2 Cosgrove D M, Rosenkranz E R, Hendren W G, Bartlett J C, Stewart W J-Valvuloplasty for aortic insufficiency. $J$ Thorac Cardiovasc Surg 1991; 102: 571-7.

3 Garamella J J, Andersen J G, Oropeza R, Veloso A, Naidu R - The surgical treatment of aortic insufficiency by open plastic revision of the tricuspid aortic valve to a bicuspid valve. Surg Gynec \& Obst 1958; 106: 679-84.

4 Frater R W - Aortic valve insufficiency due to aortic dilatation: correction by sinus rim adjustment. Circulation 1986; 47(Suppl I): I-136-42.

5 Obadia J F, Tatou E, Lançon M H, Raoux R, Brenot R, David M - Les insuffisances aortiques post-traumatiques. Arch Mal Coeur 1991; 85: 211-4.

6 Arom $\mathrm{K} V$, Nicoloff D M, Kersten T E, Lindsay W G, Northrup W F - St Jude medical prosthesis: valve related deaths and complications. Ann Thorac Surg 1987; 43: 591-8.

7 Blackstone $\mathrm{E} \mathrm{H}$ \& Kirklin J W - Death and other time related events after valve replacement. Circulation 1985; 72: 753-67.

8 Duran C, Kumar N, Gometza B, al Halees Z - Indications and limitations of aortic valve reconstruction. Ann Thorac Surg 1991; 52: 447-53.

9 Konstantinov B A, Dzemeshkevich S L, Ivanov V A, Sandrikov V A, Rikunov J - Long-term results of plastic reconstruction in acquired aortic valvular disease. $J$ Cardiovasc Surg 1994; 35 (Suppl 1): 125-8.

10 Starzl T E, Cruzat E P, Walker B A, Lewis F J - A technique for bicuspidization of the aortic valve. Thorac Cardiovasc Surg 1959; 38: 262-70.

11 Hurwitt E S, Hoffert P W, Rosemblatt A - Plication of the aortic ring in the correction of aortic insufficiency. Thorac Cardiovasc Surg 1960; 39: 655-62.

12 Cabrol C, Cabrol A, Guiraudon G, Bertrand M - Le traitement de l'insuffisance aortique par l'annuloplastie aortique. Arch Mal Coeur Vaiss 1966; 59: 1305-12. 